\title{
A SIMILAR FLOW BETWEEN TWO ROTATING DISKS*
}

\author{
BY \\ E. A. HAMZA ${ }^{\dagger}$ AND D. A. MACDONALD \\ University of Liverpool
}

\begin{abstract}
When viscous incompressible fluid is contained between two parallel disks which, at time $t$, are spaced a distance $H \sqrt{1-\alpha t}$ apart and are rotating with angular velocities proportional to $\Omega_{1}(1-\alpha t)^{-1}$ the governing Navier-Stokes equations reduce to a set of ordinary differential equations. We present approximate solutions to these equations for a range of values of the three linearly independent parameters which influence the fluid motion. Special attention is given to the normal forces and the torques which the fluid exerts on the rotating surfaces.
\end{abstract}

1. Introduction. The steady motion of viscous fluid contained between two rotating disks spaced a fixed distance $H$ apart is of especial interest in the theory of incompressible flow, not least because of a simple, yet remarkable, transformation which reduces the NavierStokes equations to a set of ordinary differential equations. This transformation was first described by T. V. Kármán [1] who studied the steady motion of viscous fluid in the semi-infinite region bounded by a single rotating disk. That the transformation applies equally well when the fluid is contained between two parallel rotating disks was first recognized by Batchelor [2]. The considerable simplification which has resulted from the replacement of the original set of partial differential equations by a set of ordinary differential equations has made possible an in depth numerical study of a three dimensional viscous flow problem which has proved to be both difficult and controversial (see, for example, Batchelor [2], Stewartson [3], Holodniok et al. [4]).

When the disks rotate with time dependent angular velocities, $\omega_{1}(t)$ and $\omega_{2}(t)$, Kármán's transformation is again applicable, but now the Navier-Stokes equations reduce to a pair of coupled non-linear partial differential equations which have been examined in the special case of impulsively started disks by Pearson [5]. It is not difficult to show that when the disks are at a fixed distance apart it is impossible to find angular velocities $\omega_{1}(t)$, $\omega_{2}(t)$ which will allow these partial differential equations to be further transformed to ordinary differential equations. But if the disks be allowed to have a velocity component in a direction perpendicular to their planes it can be shown that when their distance of

* Received February 8, 1983. First author's permanent address: School of Mathematical Sciences, University of Khartoum, P. O. Box 321, Khartoum, Sudan. 
separation at time $t$ is proportional to $H(1-\alpha t)^{1 / 2}$, where $H$ and $\alpha^{-1}$ respectively denote a representative length and a representative time, and when their angular velocities are proportional to $\Omega_{1}(1-\alpha t)^{-1}$, where $\Omega_{1}$ denotes a representative angular velocity, the partial differential equations can be reduced to a coupled pair of ordinary differential equations. These equations will be used to examine the nature of the flow field for a range of values of the non-dimensional parameters $\alpha H^{2} / 2 \nu, \Omega_{1} / \alpha, \Omega_{2} / \Omega_{1}$, where $\Omega_{1}(1-\alpha t)^{-1}$ and $\Omega_{1}(1-\alpha t)^{-1}$ denote the angular velocities of the disks. ${ }^{1}$ In particular, when $\Omega_{1} / \alpha \gg 1$ the time scale for the rate of approach (or separation) of the disks is much greater than the time scale for their rotation and the flow is then approximately that which is obtained when the disks rotate with constant angular velocities and are spaced a fixed distance apart. Also, by selecting suitable values of $\Omega_{2} / \Omega_{1}$ in the range $-1 \leqslant \Omega_{2} / \Omega_{1} \leqslant 1$ it is possible to study the differing flow regimes corresponding to disks which rotate in the same direction and disks which rotate in opposite directions.

The parameter $\Omega_{1} / \alpha$ is equal to the ratio $\left(R_{e}^{R} / 2 R_{e}^{s}\right)$, where $R_{e}^{R}=\Omega_{1} H^{2} / \nu$ is a Reynolds number based on the speed of rotation of the disks and $R_{e}^{s}=\alpha H^{2} / 2 \nu$ is a Reynolds number based on their speed of approach. The special case corresponding to $R_{e}^{R}=0$ has been examined by Ishizawa [6] and by Wang [7] and the normal forces which the fluid exerts on the disks have been evaluated; we shall examine the way in which the normal forces are modified by rotation of the disks. For $R_{e}^{R} \gg R_{e}^{s}$ the torque which the fluid exerts on the disks is of greater physical interest than is the normal force; in this instance we shall therefore examine the way in which the torque is modified by normal motion of the disks.

2. The ordinary differential equations. We consider axisymmetric incompressible flow between two parallel infinite disks which rotate in their own planes and are spaced a distance $d(t)$ apart, where $t$ denotes time. We select cylindrical polar coordinates $r, \theta, z$ and define the positions of the disks at time $t$ by $z=0$ and $z=d(t)$; the velocity components in the directions of increase of $r, \theta, z$ are taken to be $u, v$ and $w$, respectively. On the assumption that

$$
w=-2 F(z, t)
$$

we find from the mass conservation equation that

$$
u=r \partial F / \partial z \text {. }
$$

Also, the equation for the axial pressure gradient, $\partial p / \partial z$, leads to

$$
\partial^{2} p / \partial r \partial z=0
$$

and differentiation with respect to $z$ of the equation for the radial pressure gradient shows that

$$
v / r=G(z, t),
$$

where $G$ is related to $F$ by the non-linear equation

$$
F_{z z t}-2 F F_{z z z}-2 G G_{z}=\nu F_{z z z z} \text {. }
$$

\footnotetext{
'We assume, without loss of generality, that $0 \leqslant\left|\Omega_{2}\right| \leqslant \Omega_{1}$.
} 
The remaining Navier-Stokes equation, which is independent of $p$, now gives

$$
G_{t}+2 G F_{z}-2 F G_{z}=\nu G_{z z} .
$$

To search for a similarity solution we make the change of variables

$$
\xi=t, \quad \eta=z / d(t) .
$$

Equation (2.4) transforms to

$$
\frac{1}{d} F_{\eta \eta \xi}-\frac{2 d^{\prime}}{d^{2}}\left(F_{\eta \eta}+\frac{\eta}{2} F_{\eta \eta \eta}\right)-\frac{2 F}{d^{2}} F_{\eta \eta \eta}-2 G G_{\eta}=\frac{\nu}{d^{3}} F_{\eta \eta \eta \eta} .
$$

This equation will reduce to an ordinary differential equation when

$$
F=\frac{1}{d(\xi)} \times \text { function of } \eta, \quad G=\frac{1}{d^{2}(\xi)} \times \text { function of } \eta, \quad d^{\prime}=\text { constant } / d(\xi) .
$$

We choose

$$
d(\xi)=H \sqrt{1-\alpha \xi},
$$

where $\alpha$ is a constant with dimensions $t^{-1}$. Equation (2.6) now transforms to

$$
\frac{1}{R_{e}^{s}} f^{\prime \nu}=3 f^{\prime \prime}+[\eta-2 f] f^{\prime \prime \prime}-8\left(\frac{\Omega_{1}}{\alpha}\right)^{2} g g^{\prime}
$$

where

$$
F(\xi, \eta)=\frac{\alpha H}{2 \sqrt{1-\alpha \xi}} f(\eta), \quad G(\xi, \eta)=\frac{\Omega_{1}}{(1-\alpha \xi)} g(\eta), \quad R_{e}^{s}=\frac{\alpha H^{2}}{2 \nu},
$$

and $\Omega_{1}$ is a constant with the dimensions of $t^{-1}$. With this choice of $F$ and $G$ we find that Eq. (2.5) transforms to

$$
\frac{1}{R_{e}^{s}} g^{\prime \prime}=2 g+\eta g^{\prime}+2 g f^{\prime}-2 f g^{\prime}
$$

Boundary conditions. The boundary conditions for the integration of equations (2.7), (2.8) follow from the conditions:

$$
\begin{gathered}
u=0, \quad v=\frac{\Omega_{1} r}{(1-\alpha t)}, \quad w=0, \quad \text { on } z=0 \\
u=0, \quad v=\frac{\Omega_{2} r}{(1-\alpha t)}, \quad w=\frac{-\alpha H}{2 \sqrt{1-\alpha t}}, \quad \text { on } z=H \sqrt{1-\alpha t} .
\end{gathered}
$$

Thus

$$
\begin{aligned}
& f(0)=0, \quad f^{\prime}(0)=0, \quad g(0)=1, \\
& f(1)=\frac{1}{2}, \quad f^{\prime}(1)=0, \quad g(1)=s \text {, }
\end{aligned}
$$

where $s=\Omega_{2} / \Omega_{1}$.

We note that the initial conditions which are relevant to this solution are that

$$
F=\frac{\alpha H}{2} f\left(\frac{z}{H}\right), \quad G=\Omega_{1} g\left(\frac{z}{H}\right),
$$


where, for fixed $\Omega_{i} / \alpha, R_{e}^{s}$ and $s, f(\eta), g(\eta),(\eta \in[0,1])$, denote the solutions to Eqs. (2.7)-(2.10).

$2.1 \mathrm{~A}$ form of the equations suitable when $\left(\Omega_{1} / \alpha\right) \gg 1$. In the limiting case when $\left(\alpha / \Omega_{1}\right) \rightarrow 0$ Eqs. (2.7)-(2.10) do not at once reduce to the equations which govern steady flow between two rotating disks at a fixed distance apart. To obtain a form of the equations which is suitable when $0<\alpha / \Omega_{1} \ll 1$ we transform $f$ and $g$ in Eqs. (2.7) and (2.8) according to

$$
\begin{aligned}
& f=\lambda \mathfrak{F}+\eta^{2}\left(\frac{3}{2}-\eta\right), \\
& g=\mathbb{S},
\end{aligned}
$$

where $\lambda=R_{e}^{R} / R_{e}^{s}$. Equations (2.7)-(2.10) now become:

$$
\begin{gathered}
\mathfrak{F}^{\prime 0}=-2 R_{e}^{R}\left\{\mathfrak{F} \mathfrak{F}^{\prime \prime \prime}+\mathbb{S} \mathscr{S}^{\prime}\right\}+R_{e}^{s}\left[\eta(1-\eta)(1-2 \eta) \mathfrak{F}^{\prime \prime \prime}+3 \mathfrak{F}^{\prime \prime}+12 \mathfrak{F}\right] \\
+3(1-2 \eta)\left[3-2 \eta+2 \eta^{2}\right]\left(R_{e}^{s}\right)^{2} / R_{e}^{R} ; \\
\mathfrak{S}^{\prime \prime}=2 R_{e}^{R}\left[\mathbb{S} \mathfrak{F}^{\prime}-\mathfrak{F} \mathfrak{S}^{\prime}\right]+R_{e}^{s}\left[2 \mathbb{S}\left(1+3 \eta-3 \eta^{2}\right)+\eta \mathfrak{S}^{\prime}(1-\eta)(1-2 \eta)\right] ; \\
\mathfrak{F}(0)=\mathfrak{F}^{\prime}(0)=0, \quad \mathfrak{F}(1)=\mathfrak{F}^{\prime}(1)=0 ; \\
\mathbb{S}(0)=1 ; \quad \mathbb{S}(1)=s .
\end{gathered}
$$

We observe that when $\left(\alpha / \Omega_{1}\right) \rightarrow 0$ Eqs. (2.11) to (2.14) reduce to the equations which govern flow between two rotating disks spaced a fixed distance $H$ apart (in the limiting case the axial and azimuthal velocity components are given by $w=-2 \Omega_{1} H \mathcal{F}$ and $v=r \Omega_{1} g$ respectively). To examine the effect of slight squeezing on the otherwise steady flow between two rotating disks at a fixed distance apart we must compare (S) and $G_{s}$ and $\mathfrak{F}+R_{e}^{s} / R_{e}^{R} \eta^{2}\left(\frac{3}{2}-\eta\right)$ and $F_{s}$, where $G_{s}$ and $F_{s}$ denote the solutions to Eqs. (2.11)-(2.14) when $R_{e}^{s}=0$.

3. The forces which act on the disks. We shall examine the forces which act on the disks on the assumption that each is of finite radius $a$ and of negligible thickness. When $0<\Omega_{1} / \alpha<1$ the normal forces will be of greater interest than the torques whereas when $\Omega_{1} / \alpha \gg 1$ the reverse will be true.

3.1 The normal force. From the result $\partial^{2} p / \partial \eta \partial r=0$ it can be shown that $\partial p / \partial r$ may be taken in the form

$$
\frac{1}{\rho r} \frac{\partial p}{\partial r}=\frac{\alpha^{2}\left[f^{\prime \prime \prime}(0)+4 R_{e}^{s}\left(\Omega_{1} / \alpha\right)^{2}\right]}{4 R_{e}^{s}(1-\alpha t)^{2}} .
$$

Also, for a finite upper disk of radius $a$ the normal force, or load, $W$, which the fluid exerts on the disk is given by

$$
W=2 \pi\left[\int_{0}^{a} r P(r, 1, t) d r-\int_{0}^{a} r P^{*}(r, 1, t) d r\right],
$$

where $P(r, 1, t)=p(r, 1, t)-p(a, 1, t)$ and similarly for $P^{*}(r, 1, t)$ (the star notation refers to conditions on the upper side of the disk and $p(a, 1, t)$ denotes the pressure at the edge of the disk at time $t)$. We assume that $\partial p^{*}(r, 1, t) / \partial r=0$, in which case the second 
integral in (3.2) is zero. The assumption that $\partial p / \partial r$ is of the form (3.1) when the disks are of finite radius now leads to

$$
W=\frac{-\pi \mu \alpha a^{4}}{8 H^{2}(1-\alpha t)^{2}}\left[f^{\prime \prime \prime}(0)+4\left(\frac{\Omega_{1}}{\alpha}\right)^{2} R_{e}^{s}\right]
$$

or

$$
\bar{W}=-\frac{1}{6 R_{e}^{s}}\left[f^{\prime \prime \prime}(0)+4\left(\frac{\Omega_{1}}{\alpha}\right)^{2} R_{e}^{s}\right]
$$

where

$$
\bar{W}=\frac{8 W(1-\alpha t)^{2}}{3 \pi \rho \alpha^{2} a^{4}} .
$$

As the similar solution is derived under the assumption that the disks are of infinite radius the result described by (3.3) is not exact. However, the approximation will be acceptable when the diffusion distance $\sqrt{\nu t} \ll a$.

3.2 The torques exerted on the disks. When $\sqrt{\nu t} \ll a$ the torque, $T_{u}$, which the fluid exerts on the upper disk is given by

$$
T_{u}=\left.\int_{0}^{a} 2 \pi r^{2} \mu \frac{\partial v}{\partial z}\right|_{z=d(t)} d r=\frac{\pi a^{4} \Omega_{1} \mu}{2 H(1-\alpha t)^{3 / 2}} g^{\prime}(1)
$$

or, with an obvious notation,

$$
\bar{T}_{u}=g^{\prime}(1) .
$$

For the lower disk the corresponding result is

$$
\bar{T}_{L}=g^{\prime}(0) \text {. }
$$

4. Approximate solution when $R_{e}^{s}=o(1)$ and $R_{e}^{R}=o(1)$. The term $\left(\Omega_{1} / \alpha\right)^{2}$ in Eq. (2.7) is equal to $\left(R_{e}^{R} / 2 R_{e}^{s}\right)^{2}$. Therefore, we must solve the equations

$$
\begin{aligned}
& f^{\prime v}=R_{e}^{s}\left[3 f^{\prime \prime}+(\eta-2 f) f^{\prime \prime \prime}-2\left(R_{e}^{R} / R_{e}^{s}\right) g g^{\prime}\right], \\
& g^{\prime \prime}=R_{e}^{s}\left[2 g+\eta g^{\prime}+2 g f^{\prime}-2 f g^{\prime}\right],
\end{aligned}
$$

subject to the conditions (2.9) and (2.10). We describe approximate analytic solutions which are valid when $R_{e}^{R}=o(1)$ and $R_{e}^{s}=o(1)$.

When $R_{e}^{s}=o(1)$ and $R_{e}^{R}=o\left(R_{e}^{s}\right)$ the radial and axial velocity profiles and the normal forces which are exerted by the fluid on the disks are approximately equal to the values they assume when rotation of the disks is absent. This case has been studied by Ishizawa [6] and by Wang [7].

When $R_{e}^{R}=O\left(R_{e}^{s}\right)$ and when $R_{e}^{R}=O\left(R_{e}^{s}\right)^{1 / 2} f$ and $g$ may, for $R_{e}^{s}=o(1)$, be expanded in the form

$$
\begin{aligned}
& f=f_{0}+R_{e}^{s} f_{1}+O\left(R_{e}^{s}\right)^{2}, \\
& g=g_{0}+R_{e}^{s} g_{1}+O\left(R_{e}^{s}\right)^{2},
\end{aligned}
$$


where $f_{0}, f_{1}, g_{0}, g_{1}$ satisfy the conditions:

$$
\begin{array}{ll}
f_{0}(0)=f_{0}^{\prime}(0)=0, & f_{0}(1)=\frac{1}{2}, \quad f_{0}^{\prime}(1)=0 ; \\
f_{1}(0)=f_{1}^{\prime}(0)=0, & f_{1}(1)=f_{1}^{\prime}(1)=0 ; \\
g_{0}(0)=1, & g_{0}(1)=\Omega_{2} / \Omega_{1} \equiv s ; \\
g_{1}(0)=0, & g_{1}(1)=0 .
\end{array}
$$

Case (i): $R_{e}^{R}=O\left(R_{e}^{s}\right)$. In this case $f_{0}, g_{0}, f_{1}, g_{1}$, satisfy the equations

$$
\begin{aligned}
& f_{0}^{\prime \nu}=0, \quad g_{0}^{\prime \prime}=0, \\
& f_{1}^{\prime \nu}=3 f_{0}^{\prime \prime}+\left(\eta-2 f_{0}\right) f_{0}^{\prime \prime \prime}-2\left(\frac{R_{e}^{R}}{R_{e}^{s}}\right)^{2} g_{0} g_{0}^{\prime}, \\
& g_{1}^{\prime \prime}=2 g_{0}+\eta g_{0}^{\prime}+2 g_{0} f_{0}^{\prime}-2 f_{0} g_{0}^{\prime} .
\end{aligned}
$$

We obtain

$$
\begin{aligned}
f_{0}= & \eta^{2}\left(\frac{3}{2}-\eta\right), \quad g_{0}=1-(1-s) \eta, \\
f_{1}= & \eta^{2}(1-\eta)^{2}\left[\frac{19}{280}-\frac{1}{7} \eta+\frac{3}{140} \eta^{2}-\frac{1}{70} \eta^{2}\right. \\
& \left.-\frac{(1-s)^{2}}{60}\left(\frac{R_{e}^{R}}{R_{e}^{s}}\right)^{2}(2+\eta)+\frac{(1-s)}{12}\left(\frac{R_{e}^{R}}{R_{e}^{s}}\right)^{2}\right], \\
g_{1}= & \frac{1}{20} \eta(1-\eta)\left[-(11 s+19)+(1-11 s) \eta+(11-s) \eta^{2}-4(1-s) \eta^{3}\right],
\end{aligned}
$$

where the parameter $s\left(\equiv \Omega_{2} / \Omega_{1}\right)$ may, by symmetry, be taken to lie in the interval $-1 \leqslant s \leqslant 1$.

Case (ii): $R_{e}^{R}=O\left(R_{e}^{s}\right)^{1 / 2}$. The equations satisfied by $f_{0}, g_{0}, f_{1}, g_{1}$ are now:

$$
\begin{aligned}
& f_{0}^{\prime v}=-2\left(\left(R_{e}^{R}\right)^{2} / R_{e}^{s}\right) g_{0} g_{0}^{\prime}, \quad g_{0}^{\prime \prime}=0, \\
& f_{1}^{\prime v}=3 f_{0}^{\prime \prime}+\left(\eta-2 f_{0}\right) f_{0}^{\prime \prime \prime}-2\left(R_{e}^{R}\right)^{2} / R_{e}^{s}\left[g_{0} g_{1}^{\prime}+g_{0}^{\prime} g_{1}\right] \\
& g_{1}^{\prime \prime}=2 g_{0}+\eta g_{0}^{\prime}+2 g_{0} f_{0}^{\prime}-2 f_{0} g_{0}^{\prime} .
\end{aligned}
$$

We obtain

$$
\begin{aligned}
& f_{0}=\eta^{2}\left(\frac{3}{2}-\eta\right)+\frac{1}{60}\left(R_{e}^{R}\right)^{2} / R_{e}^{s}(1-s) \eta^{2}(1-\eta)^{2}\{5-(2+\eta)(1-s)\} \\
& g_{0}=1-(1-s) \eta
\end{aligned}
$$

The expressions for $f_{1}$ and $g_{1}$, which are more lengthy than in case (i), will not be quoted here.

4.1 The load and the torques. By use of Eqs. (3.3) and (3.4) the forces necessary to maintain the disks in motion with the velocities specified in Sec. 2 may be calculated when $R_{e}^{s}=o(1)$ and $R_{e}^{R}=O\left(R_{e}^{s}\right)$, or $R_{e}^{R}=O\left(R_{e}^{s}\right)^{1 / 2}$. 
Case (i): $R_{e}^{R}=O\left(R_{e}^{s}\right)$. When $R_{e}^{R}=O\left(R_{e}^{s}\right)$ the results for $\bar{W}, \bar{T}_{u}$ and $\bar{T}_{L}$ are given by

$$
\begin{aligned}
& \bar{W}=\frac{1}{R_{e}^{s}}\left[1-\frac{R_{e}^{s}}{6}\left\{-\frac{117}{70}+\left(\frac{R_{e}^{R}}{R_{e}^{s}}\right)^{2}\left[\frac{3}{10}(1-s)^{2}+s\right]\right\}+O\left(R_{e}^{s}\right)^{2}\right], \\
& \bar{T}_{u}=-1+s+\frac{R_{e}^{s}}{20}\{19 s+11\}+O\left(R_{e}^{s}\right)^{2}, \\
& \bar{T}_{L}=-1+s-\frac{R_{e}^{s}}{20}\{11 s+19\}+O\left(R_{e}^{s}\right)^{2} .
\end{aligned}
$$

Case (ii): $R_{e}^{R}=O\left(R_{e}^{s}\right)^{1 / 2}$. When $R_{e}^{R}=O\left(R_{e}^{s}\right)^{1 / 2}$ we obtain

$$
\begin{aligned}
\bar{W}= & \frac{1}{R_{e}^{s}}\left[1-\frac{1}{60}\left(R_{e}^{R}\right)^{2} / R_{e}^{s}\left(3 s^{2}+4 s+3\right)\right. \\
& +\frac{R_{e}^{s}}{420}\left\{117+\frac{1}{45}\left(R_{e}^{R}\right)^{2} / R_{e}^{s}\left(521 s^{2}+983 s+521\right)\right. \\
& \left.\left.+\frac{1}{10,800}\left[\frac{\left(R_{e}^{R}\right)^{2}}{R_{e}^{s}}\right]^{2}\left[579 s^{4}-16 s^{3}-10966 s^{2}-16 s+579\right]\right\}+O\left(R_{e}^{s}\right)^{2}\right],
\end{aligned}
$$

$\left.\bar{T}_{u}=-1+s+\frac{R_{e}^{s}}{20}\left\{19 s+11+\frac{1}{315}\left(R_{e}^{R}\right)^{2} / R_{e}^{s}\left(27 s^{3}-11 s^{2}-24 s+8\right)\right\}+O\left(R_{e}^{s}\right)^{2}\right]$,

$\left.\bar{T}_{L}=-1+s+\frac{R_{e}^{s}}{20}\left\{-11 s-19-\frac{1}{315}\left(R_{e}^{R}\right)^{2} / R_{e}^{s}\left(8 s^{3}-24 s^{2}-11 s+27\right)\right\}+O\left(R_{e}^{s}\right)^{2}\right]$.

4.2 Observations from the perturbation solution. In the special case when $\Omega_{1}=\Omega_{2}=0$ the radial velocity component is everywhere positive. Hence, the radial pressure gradient, which is independent of $z$, is negative. When the lower disk rotates but the upper does not the stress distribution near each disk is modified and the radial outflow from the lower disk $(z=0)$ is greater than that for the case when $\Omega_{1}=0$, whereas the outflow from the upper disk is less. This reduced radial outflow near $z=1$ results from an increased radial pressure gradient, which is necessary by virtue of the requirement that mass conservation must be satisfied. Indeed, for sufficiently large $\left(R_{e}^{R} / R_{e}^{s}\right)$ the radial pressure gradient will be positive and the radial flow near the upper disk will be inwardly directed (the extent of this region of inward radial flow will depend on the magnitude of $R_{e}^{R} / R_{e}^{s}$ ).

When both disks rotate, so that all three of $R_{e}^{R}, R_{e}^{s}$ and $s$ are nonzero, it is possible to have an outwardly directed radial flow near both disks with an inwardly directed radial flow, which is driven by the positive radial pressure gradient, elsewhere.

The velocity field. The radial velocity component is described by the dimensionless function $f^{\prime}(\eta)$ which, for $R_{e}^{R}=O\left(R_{e}^{s}\right)^{1 / 2}$, is given by

$$
\begin{aligned}
f^{\prime}(\eta)= & \eta(1-\eta)\left[3+\frac{1}{60}\left(R_{e}^{R}\right)^{2} / R_{e}^{s}(1-s)\{10(1-2 \eta)\right. \\
& \left.\left.+(1-s)\left(5 \eta^{2}+5 \eta+4\right)\right\}\right]+O\left(R_{e}^{s}\right) .
\end{aligned}
$$


We observe from (4.5) that when $s>-\frac{2}{3}$ and when

$$
\left(R_{e}^{R}\right)^{2} / R_{e}^{s}>\frac{90}{(1-s)(2+3 s)}
$$

the leading term in the expansion for $f^{\prime}(\eta)$ will be negative near $\eta=1$.

The radial pressure gradient is given by the equation

$$
\frac{1}{\rho r} \frac{\partial p}{\partial r}=\frac{\alpha^{2}}{4 R_{e}^{s}(1-\alpha t)^{2}}\left[-6+\frac{1}{10}\left(R_{e}^{R}\right)^{2} / R_{e}^{s}\left(3+4 s+3 s^{2}\right)+O\left(R_{e}^{s}\right)\right] \text {. }
$$

Hence, when condition (4.6) is satisfied, the leading term in the expansion for $\partial p / \partial r$ satisfies

$$
\frac{1}{\rho r} \frac{\partial p}{\partial r}>\frac{15 \alpha^{2}}{4 R_{e}^{s}(1-\alpha t)^{2}}\left[\frac{3 s^{2}+2 s+1}{(1-s)(2+3 s)}\right] .
$$

The smallest value of $\left(R_{e}^{R}\right)^{2} / R_{e}^{s}$ satisfying (4.6) occurs when $s=1 / 6$ and is $216 / 5$. Clearly, at these values of $\left(R_{e}^{R}\right)^{2} / R_{e}^{s}$ and $s \partial p / \partial r>0$, which condition is necessary (but not, as can be seen from (4.7), sufficient) if fluid near the upper disk is to move radially inwards.

When $s=-1 f^{\prime}(\eta)$ is symmetrical about the mid-plane $\eta=1 / 2$. Fluid near the disks is thrown radially outwards by centrifugal action and, for sufficiently large $\left(R_{e}^{R} / R_{e}^{s}\right)$, is drawn inwards in the vicinity of the mid-plane $\eta=1 / 2$, the driving force being the positive radial pressure gradient. Examination of Eq. (4.5) indicates that when $s=-1.0$ $f_{0}^{\prime}(1 / 2)<0$ when $\left(R_{e}^{R}\right)^{2} / R_{e}^{s}>180$. Numerical integration of Eqs. (4.1), (4.2) shows that when $R_{e}^{s}=0.01$ and $s=-1.0 f^{\prime}(1 / 2)<0$ when $\left(R_{e}^{R}\right)^{2} / R_{e}^{s}>179.6$.

For $s=0.5$ and $s=-0.5$ Eq. (4.5) predicts that $f_{0}^{\prime}\left(1^{-}\right)>0$ for $\left(R_{e}^{R}\right)^{2} / R_{e}^{s}>360 / 7$ and $\left(R_{e}^{R}\right)^{2} / R_{e}^{s}>120$, respectively. The corresponding results obtained by numerical integration of Eqs. (4.1), (4.2) when $R_{e}^{s}=0.01$ are $\left(R_{e}^{R}\right)^{2} / R_{e}^{s}>57.6$ and $\left(R_{e}^{R}\right)^{2} / R_{e}^{s}>100$, respectively.

The load. When $\left(R_{e}^{R}\right)^{2} / R_{e}^{s}=O(1)$ the non-dimensional load is given by

$$
\bar{W}=\frac{1}{R_{e}^{s}}\left[1-\frac{1}{60}\left(R_{e}^{R}\right)^{2} / R_{e}^{s}\left(3 s^{2}+4 s+3\right)+O\left(R_{e}^{s}\right)\right] \text {. }
$$

To order $R_{e}^{s}$, we note that $\bar{W}$ will decrease when $s$ and $R_{e}^{s}$ are held fixed and $R_{e}^{R}$ is increased. To explain this behaviour in physical terms we observe that an increase in the angular speed of the lower disk will, for fixed $s$ and $R_{e}^{s}$, lead to an increase in the radial outflow near $\eta=0$. Conservation of mass can be satisfied only by an increase in the radial pressure gradient. This increase in $\partial p / \partial r$ is responsible for the decrease in $\bar{W}$.

When $s$ and $R_{e}^{R}$ are held fixed differentiation of equation 4.8 with respect to $R_{e}^{s}$ predicts that when

$$
R_{e}^{s}>L, \quad L=\frac{\left(3 s^{2}+4 s+3\right)\left(R_{e}^{R}\right)^{2}}{30},
$$

$\bar{W}$ will decrease with increase of $R_{e}^{s}$, the rate of decrease being inversely proportional to $\left(R_{e}^{s}\right)^{2}$ and being influenced by $s$ and by $R_{e}^{R}$; on the other hand, $\bar{W}$ is predicted to increase 
with increase of $R_{e}^{s}$ when $R_{e}^{s}<L$. Finally, we note that for fixed $R_{e}^{R}$ and $R_{e}^{s}$ and, for $\left(R_{e}^{R}\right)^{2} / R_{e}^{s}=O(1), \bar{W}$ will attain its maximum value when $s=-2 / 3$ and for fixed $s$ $\bar{W}=O\left(R_{e}^{s}\right)$ when $R_{e}^{s}=L / 2$.

The torque. When $s=1$ the magnitude of the torque which the fluid exerts on each disk is given by

$$
\frac{3 \pi a^{4} \Omega_{1} \mu}{4 H(1-\alpha t)^{3 / 2}} R_{e}^{s}+O\left(R_{e}^{s}\right)^{2} .
$$

Hence, as $R_{e}^{s} \rightarrow 0$ the torques which act on the disks tend to zero. In this limiting case the disks rotate with constant angular velocities and the fluid contained between them is in solid body rotation. But when $R_{e}^{s} \neq 0$ and $s=1$ the angular velocities of the disks-and hence the rate of diffusion of angular momentum-are time dependent. Therefore, solid body rotation is not possible.

5. The numerical integration of the ordinary differential equations. The perturbation analysis of Sec. 4 is strictly valid only when $R_{e}^{s}=o(1)$ and $R_{e}^{R}=o(1)$. For more detailed information on the way in which the flow field is modified by changes in $R_{e}^{s}$ and $R_{e}^{R}$ it is necessary to solve the governing equations numerically.

The equations were integrated by use of the Nottingham Algorithms Group program D02 ADA, which makes efficient use of the so called shooting technique for the solution of two point boundary value problems. This is the technique which was used by Cochran [8] and by Lance and Rogers [9]. The algorithm requires the specification of initial values for unknown boundary conditions (derivations of $f$ and $g$ ) at $\eta=0$ and $\eta=1$. For $R_{e}^{s}=o(1)$ and $R_{e}^{R}=o(1)$, these were provided by the perturbation results of Sec. 4 .

In the special case when $R_{e}^{R}=0$ the results were in full agreement with those quoted by Wang [7]. Further checks on the accuracy of the numerical work were made by comparing, at fixed $R_{e}^{s}$ and $R_{e}^{R}$, the numerical results obtained from the two sets of Eqs. (4.1), (4.2) and (2.11), (2.12), and by running the computer program on a 1906s computer which retained 11 significant figures and on a CDC 7600 machine which retained fifteen.

\section{Results and discussion.}

6.1 The special case when $R_{e}^{R} \gg R_{e}^{s}$. When the Reynolds numbers $R_{e}^{R}$ and $R_{e}^{s}$ satisfy $R_{e}^{R} \gg R_{e}^{s}$ the ratio of the time scales for rotation and squeezing, $\alpha / \Omega_{1}$, satisfies $0<\alpha / \Omega_{1}$ $\ll 1$. Moreover, in the time interval $\left[t_{0}, t_{0}+\Omega_{1}^{-1}\right]$ at least one of the disks will rotate once and, when $0<\alpha t_{0} \ll 1$, the upper disk will descend a distance

$$
\int_{t_{0}}^{t_{0}+\Omega_{1}^{1}} \frac{\alpha H}{2 \sqrt{1-\alpha t}} d t=H\left[\frac{\alpha}{2 \Omega_{1}}=o\left(\frac{\alpha}{\Omega_{1}}\right)\right] .
$$

Hence, when $0<\alpha t_{0} \ll 1$ and $R_{e}^{R} \gg R_{e}^{s}$, the normal motion of the upper disk may in a first approximation be neglected. As the azimuthal velocities of the disks are proportional to $(1-\alpha t)^{-1}$, i.e. to $\left(1-\alpha\left(\Omega_{1} t\right) / \Omega_{1}\right)^{-1}$, the change in the angular velocity over a time interval of order $\Omega_{1}^{-1}$ may also be neglected, i.e. over a time scale which is small compared to $\alpha^{-1}$ the disks may be assumed to rotate with constant angular velocities. Hence, when 
$s=0.0$

\begin{tabular}{|c|c|c|c|c|c|c|c|}
\hline & & & $=10.00$ & & & 40.0 & \\
\hline$\eta$ & $R_{e}^{s}$ & $\bar{F}$ & $\frac{d \bar{F}}{d \eta}$ & (B) & $F$ & $\frac{d \bar{F}}{d \eta}$ & (B) \\
\hline & 0.0 & .0110 & .0761 & .7436 & .0256 & .1432 & .4913 \\
\hline 0.2 & 0.05 & .0112 & .0775 & .7379 & .0255 & .1427 & .4888 \\
\hline & 0.50 & .0127 & .0915 & .6925 & .0250 & .1395 & .4693 \\
\hline & 0.0 & .0221 & .0241 & .5361 & .0409 & .0071 & .3035 \\
\hline 0.4 & 0.05 & .0226 & .0269 & .5281 & .0409 & .0073 & .2990 \\
\hline & 0.50 & .0282 & .0535 & .4656 & .0403 & .0109 & .2653 \\
\hline & 0.0 & .0197 & -.0437 & .3561 & .0316 & -.0880 & .2113 \\
\hline 0.6 & 0.05 & .0210 & -.0396 & .3488 & .0317 & -.0866 & .2064 \\
\hline & 0.50 & .0330 & -.0036 & .2920 & .0327 & -.0742 & .1705 \\
\hline & 0.0 & .0079 & -.0632 & .1794 & .0115 & -.0965 & .1124 \\
\hline 0.8 & 0.05 & .010 & -.0597 & .1751 & .0120 & -.0948 & .1093 \\
\hline & 0.50 & .0289 & -.0291 & .1419 & .0159 & -.0808 & .0867 \\
\hline
\end{tabular}

TABLE 1. The effect of slight squeezing at rotation Reynolds numbers of 10.0 and 40.0 . [upper disk not rotating].

$$
\bar{F}=\mathfrak{F}+R_{e}^{s} / R_{e}^{R} \eta^{2}\left(\frac{3}{2}-\eta\right) .
$$

$\alpha>0$ and $\alpha t=o(1)$ the fluid motion between the disks must approximate that between two rotating disks spaced at a fixed distance apart.

For values of $R_{e}^{R}$ equal to 10.0 and 40.0 and for the case when the upper surface does not rotate this solution is compared in table 1 to the solution of Eqs. (2.11)-(2.14) with $R_{e}^{s}$ taking values up to 0.5 . [We note-see Sec. 2.1 - that the effect of the normal motion of the rotating disks can be guaged by examining the response of $\bar{F}=\mathfrak{F}+R_{e}^{s} / R_{e}^{R} \eta^{2}\left(\frac{3}{2}-\eta\right)$ and (S) to changes in $R_{e}^{s}$ when $s$ and $R_{e}^{R}$ are held fixed].

6.2 The velocity field. Numerical results have been obtained for squeeze Reynolds numbers $R_{e}^{s}$ which range from $o(1)$ to 10 and for rotational Reynolds numbers $R_{e}^{R}$ which range from $o(1)$ to 100 . We shall present results for two values of $R_{e}^{s}$, i.e. $R_{e}^{s}=0.01$ and $R_{e}^{s}=10.0$. For each of these values, $R_{e}^{R}$ will range form $o(1)$ to 100 and $s$ will range from -1 to +1 .

Case $R_{e}^{s}=0.01$ (slight squeezing). When $R_{e}^{R}=o(1)$ the results for $f, f_{\eta}$ and $g$ are well described by the perturbation solution of Sec. 4. In particular, when $R_{e}^{R}$ is $O\left(R_{e}^{s}\right)$, or smaller, we obtain the results

$$
f=\eta^{2}\left(\frac{3}{2}-\eta\right)+O\left(R_{e}^{s}\right), \quad g=1-(1-s) \eta+O\left(R_{e}^{s}\right) .
$$



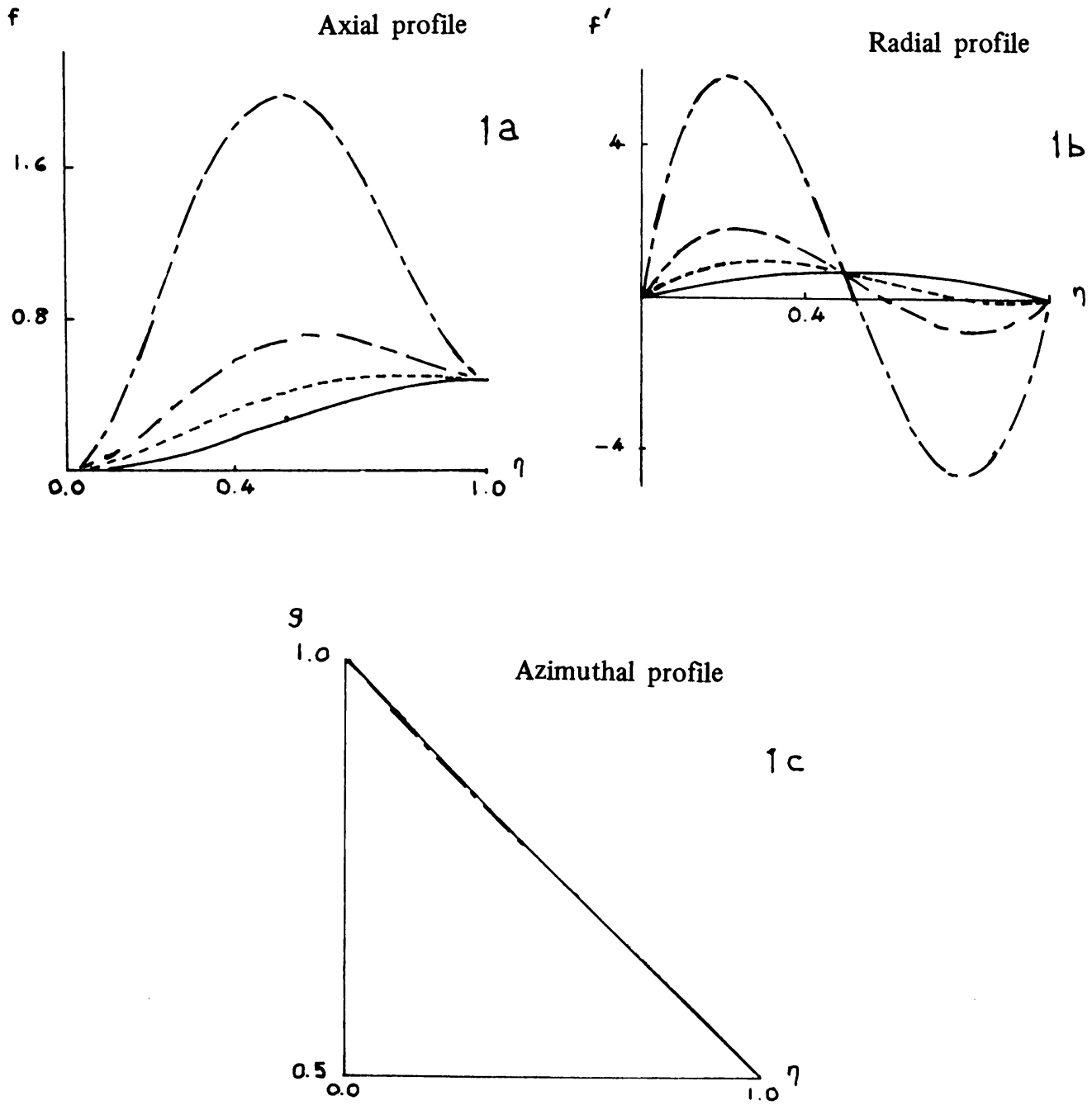

FIG. 1. The dimensionless functions $f(\eta), f^{\prime}(\eta)$ and $g(\eta)$ when $s=0.5$ and $R_{e}^{s}=0.01$. - , $R_{e}^{R}=0.02 ;-, R_{e}^{R}=0.9 ;-, R_{e}^{R}=1.5 ;-, R_{e}^{R}=3.0$.

Thus the azimuthal velocity component adjusts linearly from the value unity at $\eta=0$ to the value $s$ at $\eta=1$.

When $R_{e}^{R}=O(1)\left(R_{e}^{R} / R_{e}^{s}\right)$ is $O\left(R_{e}^{s}\right)^{-1}$ and is sufficiently large for the radial velocity component to be directed inwards, i.e. towards the axis of rotation, near the slower rotating disk at values of $s$ not too close to unity. As $s=0.5$, for example, the numerical results predict that a region of inward radial flow will exist when $R_{e}^{R}>0.76 .^{2}$ For this

${ }^{2}$ Perturbation theory gives $R_{e}^{R}>0.72$ (see Eq. (4.6)). 

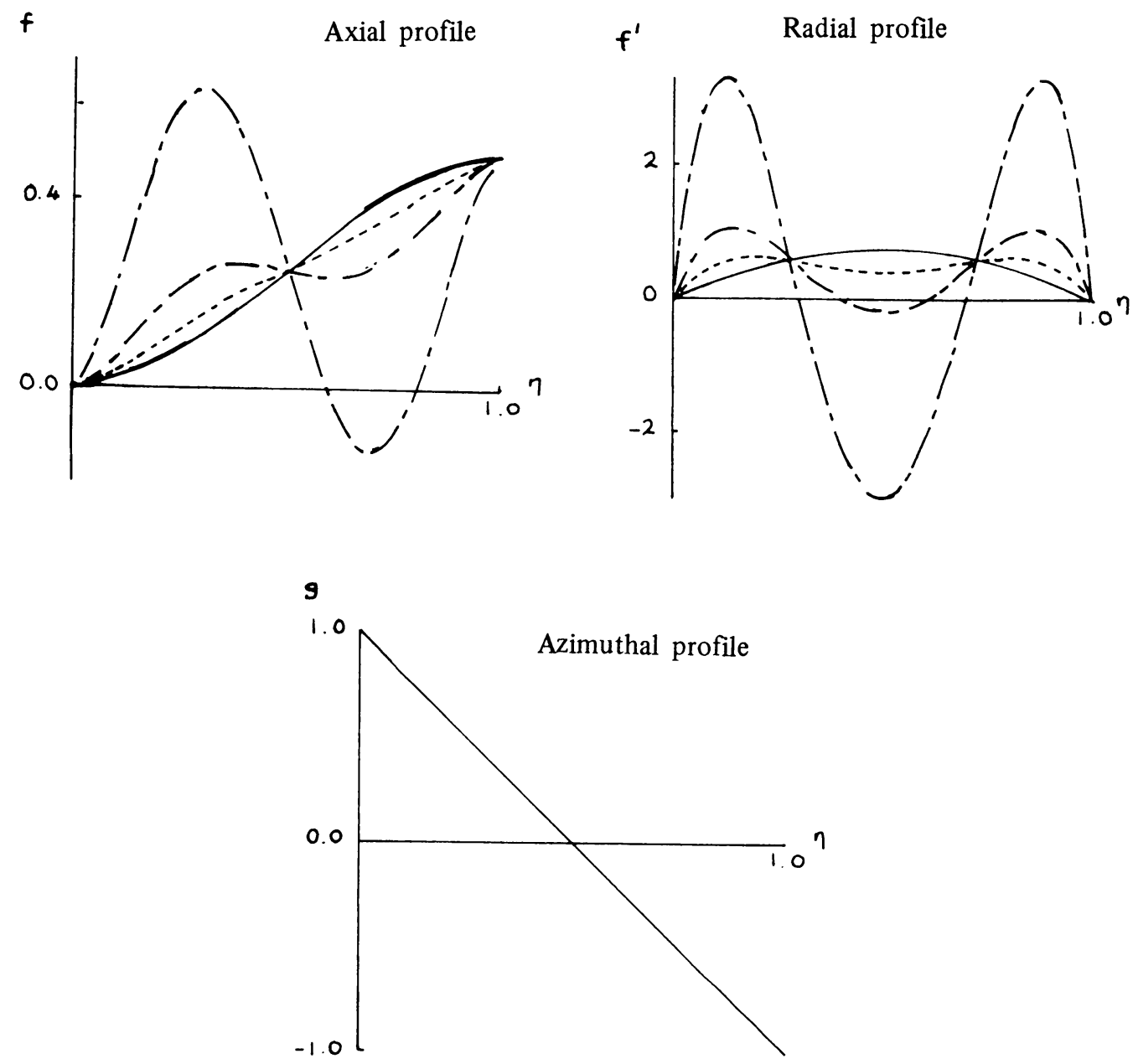

FIG. 2. The dimensionless functions $f(\eta), f^{\prime}(\eta)$ and $g(\eta)$ when $s=-1.0$ and $R_{e}^{s}=0.01$. - $R_{e}^{R}=0.02 ;-, R_{e}^{R}=0.9 ;-, R_{e}^{R}=1.5 ;-, R_{e}^{R}=3.0$.

value of $s$ and for a range of values of $R_{e}^{R}$ Fig. 1 displays the dimensionless functions $f(\eta)$, $f^{\prime}(\eta), g(\eta)$ which describe the velocity components $u, v$ and $w$. In Fig. $1 \mathrm{~b}$ regions of reversed flow are evident near $\eta=1$ when $R_{e}^{R}=0.9,1.5$ and 3.0. For the same range of $R_{e}^{R}$, Fig. 2 presents the corresponding results for $s=-1.0$, i.e. for the case of counter rotating disks. For $R_{e}^{R}>1.34$ the numerical results indicate that at this value of $s$ the radial velocity in the vicinity of the mid plane $\eta=\frac{1}{2}$ will be directed inwards towards the axis. This inwardly directed flow is evident on figure $2 \mathrm{~b}$ for $R_{e}^{R}=1.5$ and $R_{e}^{R}=3.0$. Despite appreciable changes in both $f$ and $f_{\eta}$ as $R_{e}^{R}$ varies from 0.2 to $3.0 \mathrm{~g}$, to a good order of approximation, remains linear. 

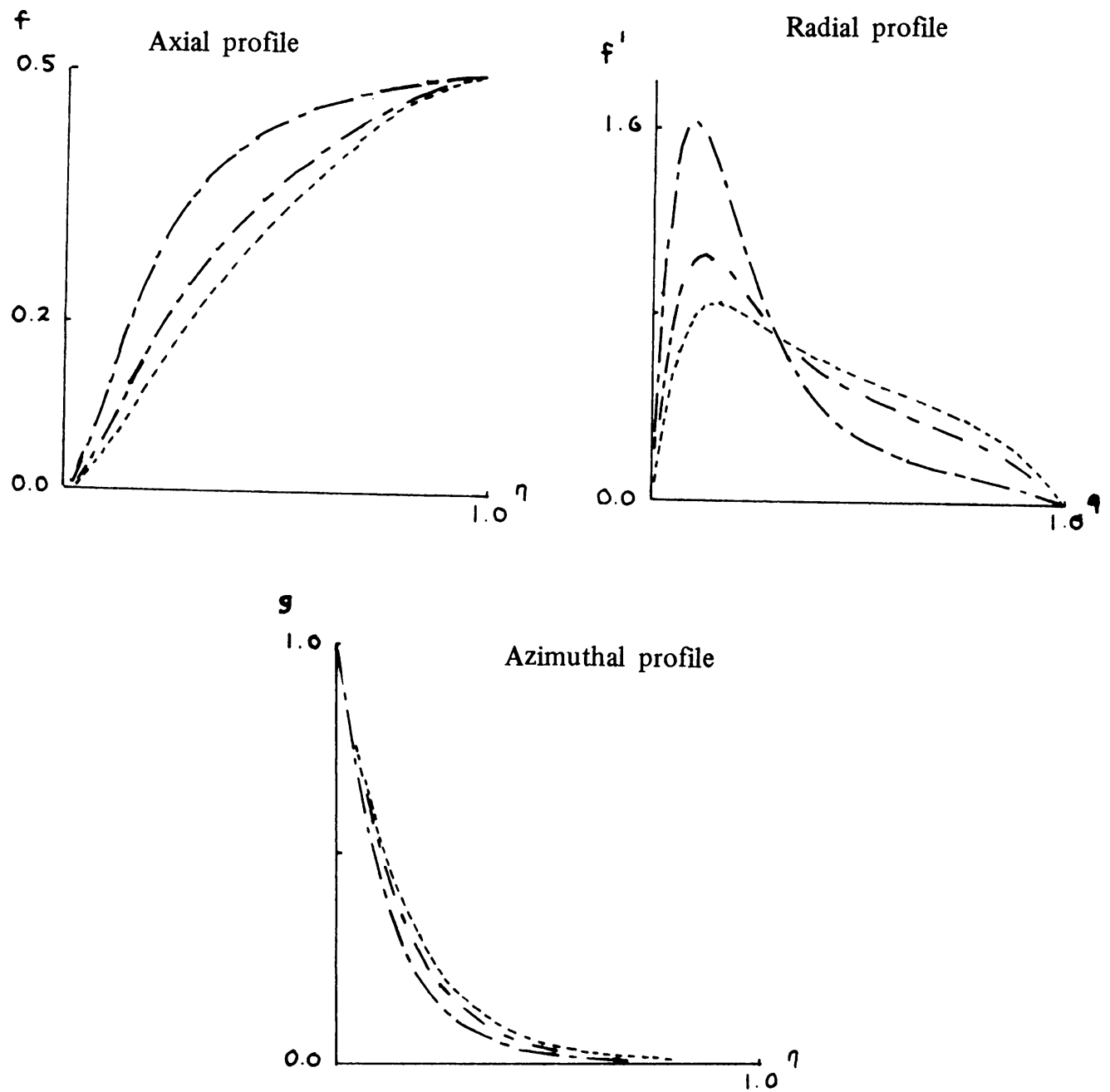

Fig. 3. The dimensionless functions $f(\eta), f^{\prime}(\eta)$ and $g(\eta)$ when $s=0.0$ and $R_{e}^{s}=10.0 .-, R_{e}^{R}=50.0 ;-, R_{e}^{R}=65.0 ;-, R_{e}^{R}=100.0$

Case $R_{e}^{s}=10.0$ (Moderate squeezing). When $R_{e}^{R} / R_{e}^{s}=o(1)$ the term $2\left(R_{e}^{R} / R_{e}^{s}\right)^{2} g g^{\prime}$ in Eq. (4.1) is negligible when compared to the other terms. Therefore, $f$ is approximately independent of $R_{e}^{R}$, as is $g$ (see Eq. (4.2)).

For the case of an upper disk which does not rotate, Fig. 3 represents the profiles of $f, f_{\eta}$ and $g$ at values of $R_{e}^{R}$ equal to 50,65 and 100 . Note the rapid rate of change of both $g$ and $f_{\eta}$ near the rotating disk, $\eta=0$. The very small value of $g$ for $\eta>0.5$ when $R_{e}^{R}=100$ is presumably due to the normal motion of the upper disk restricting the outward diffusion of angular momentum. For the case of counter rotating disks, i.e. $s=-1.0$, Fig. 4 

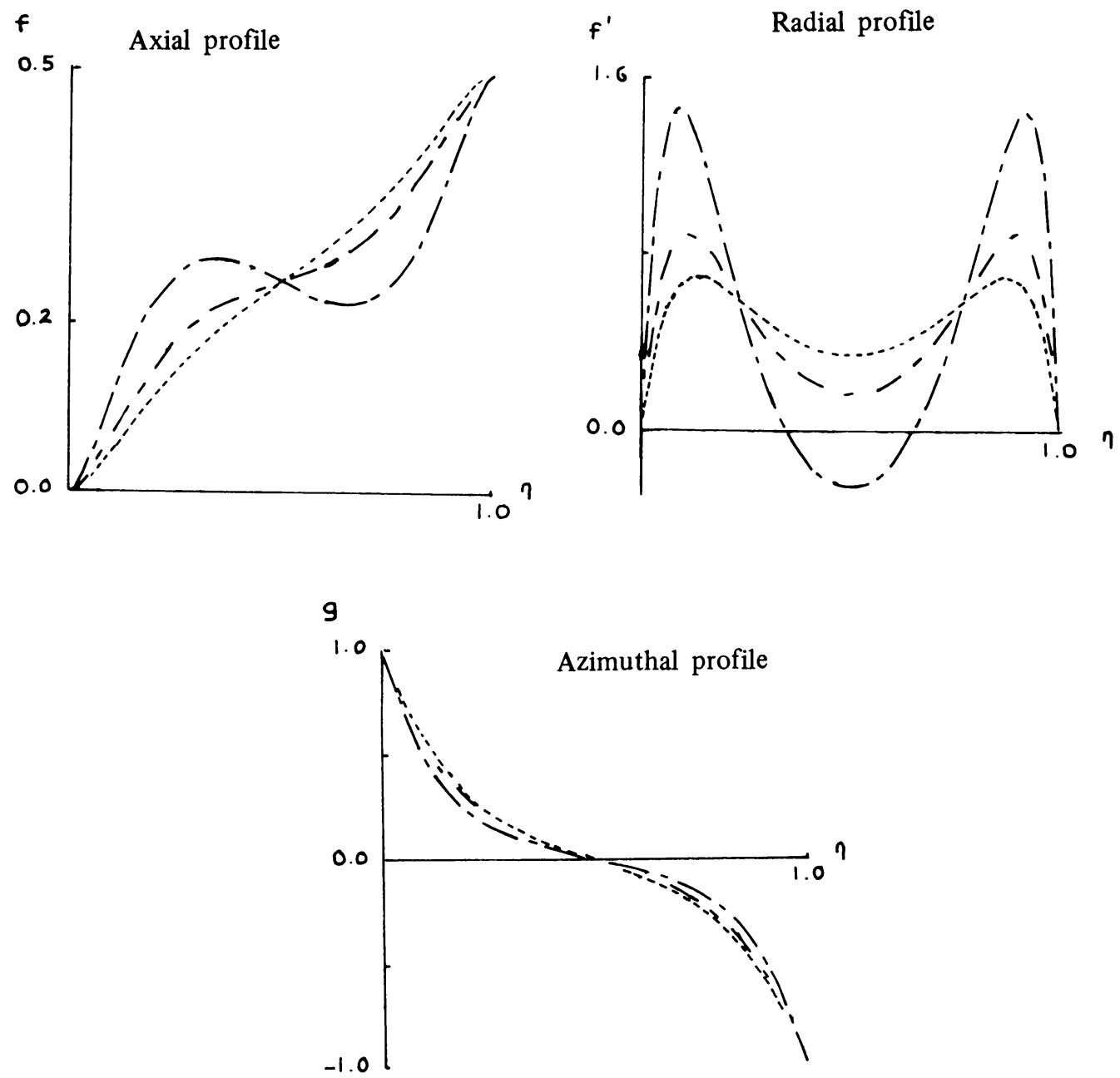

Fig. 4. The dimensionless functions $f(\eta), f^{\prime}(\eta)$ and $g(\eta)$ when $s=-1.0$ and $R_{e}^{s}=10.0 .-, R_{e}^{R}=50.0 ;-, R_{e}^{R}=65.0 ;-, R_{e}^{R}=100.0$

presents profiles of $f, f_{\eta}$ and $g$ at the same rotational Reynolds numbers. At $R_{e}^{R}=100$ the centrifugal action of the disks is sufficiently great to induce an inward radial flow in the vicinity of $\eta=0.5$.

6.3 The load. When rotation is negligible in comparison to squeezing, i.e. when $0<\left(R_{e}^{R} / R_{e}^{s}\right) \ll 1$, fluid is everywhere driven radially outwards by the negative, $z$ independent, radial pressure gradient. The normal force, or load, $\bar{W}$, which is exerted on the upper surface is then positive. But for sufficiently large values of $\left(R_{e}^{R} / R_{e}^{s}\right)$ conservation of mass is possible only when a region of inward radial flow-either in the vicinity of the slower rotating disk or, when $s= \pm 1$, in the vicinity of the mid-plane $\eta=\frac{1}{2}-$ is present. In this 

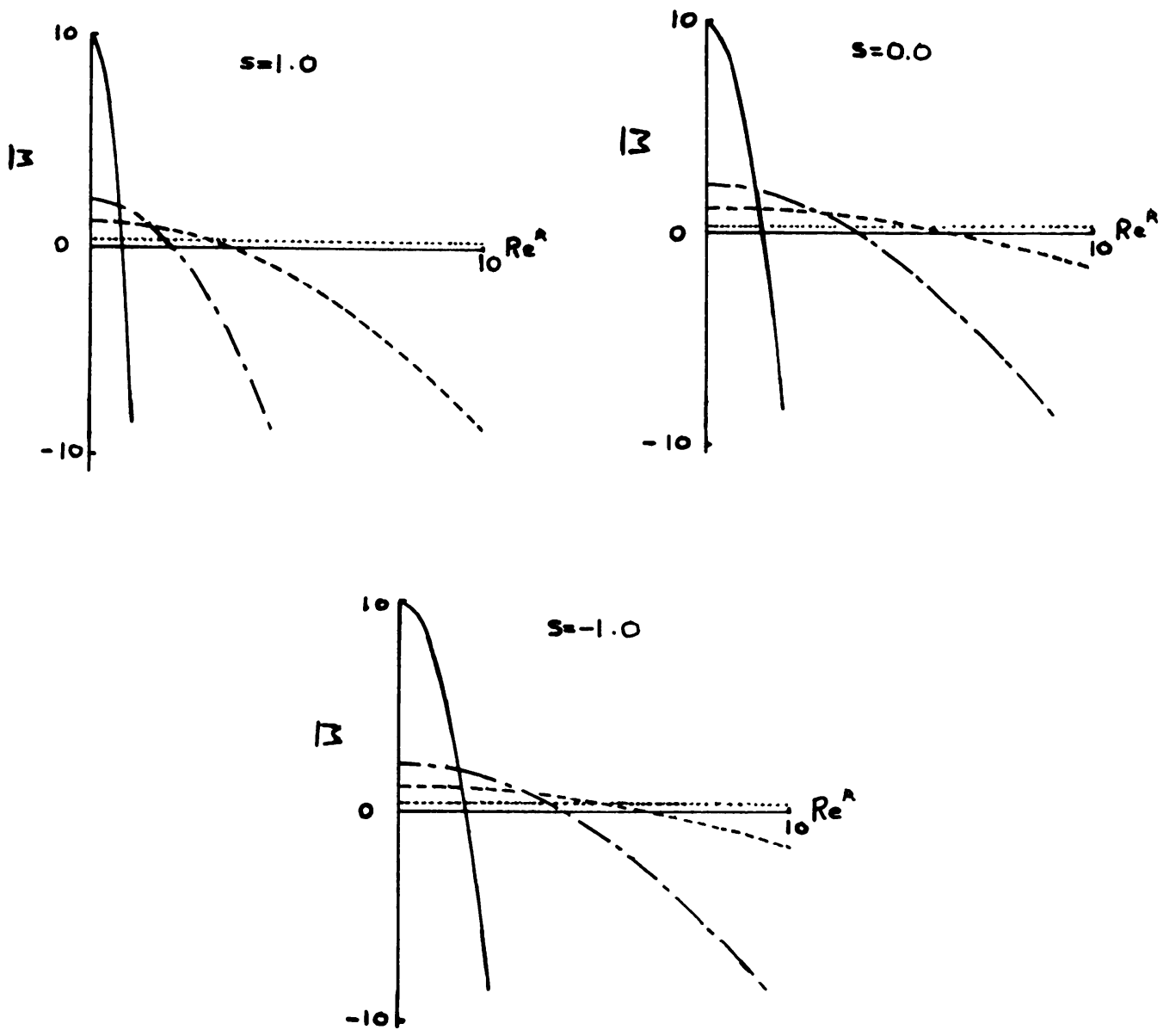

Response of the non-dimensional load, or normal force, to changes in

FIG. 5. the rotation Reynolds number $R_{e}^{R}$. -,$R_{e}^{s}=0.1 ;-, R_{e}^{s}=0.5 ;-$, $R_{e}^{s}=1.0 ;-, R_{e}^{s}=10.0$

situation it is necessary that the radial pressure gradient be positive. Therefore, $\bar{W}$ is negative. (It is also possible to have $\partial p / \partial r>0$ but to have no region of inward radial flow; in such circumstances the radial pressure gradient is nowhere sufficiently large to counteract viscous stresses which act to drive fluid radially outwards.)

Figure 5 shows, for values of $s$ equal to 0.0 and \pm 1.0 , the way in which $\bar{W}$ varies with $R_{e}^{R}$ when $R_{e}^{s}$ takes the values $0.1,0.5,1.0$ and 10.0. It is apparent from this figure that, for fixed $R_{e}^{s}, \frac{e}{W}$ decreases more rapidly with increase of $R_{e}^{R}$ when $s=1.0$ than when $s=0.0$ or -1.0 .

6.4 The torques. When $\left(R_{e}^{R} / R_{e}^{s}\right) \gg 1$ the torques which the fluid exerts on the rotating disks are of greater interest than are the normal forces. For a range of values of $R_{e}^{R}$ 

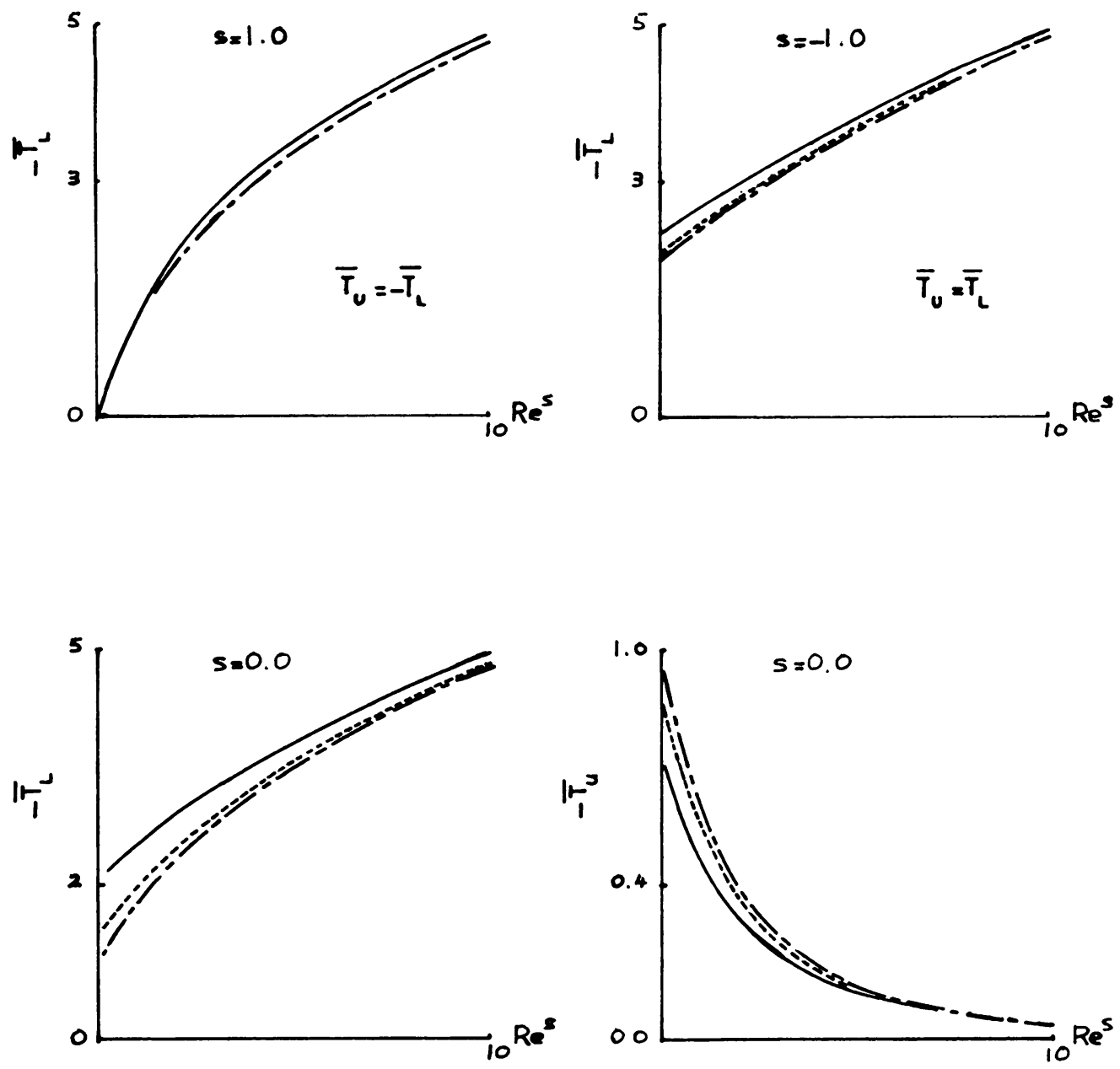

Response of the non-dimensional torques on the upper and lower disks

FIG. 6. to changes in the squeeze Reynolds number $R_{e}^{s}$. -,$R_{e}^{R}=1.0 ;-$, $R_{e}^{R}=10.0 ;-, R_{e}^{R}=20.0$

extending to $R_{e}^{R}=20$, Fig. 6 shows how the non-dimensional torques on the upper and lower disks, $\bar{T}_{u}$ and $\bar{T}_{L}$ respectively, vary with $R_{e}^{s}$. It is apparent from the figure that the torques are more sensitive to changes in $R_{e}^{s}$ than to changes in $R_{e}^{R}$. This trend is evident from the perturbation solution of Sec. 4 .

6.5 Uniqueness of the solutions. The equations governing steady flow between two rotating disks at a fixed distance apart do not have a unique solution when $R_{e}^{R}>\gamma(s)$. The dependence of $\gamma$ on $s$ is not known but Holodniok et al. [10] have shown that at $s=0.8 \gamma \sim 205$. 
For unsteady flow between the disks the situation is more complicated. Pearson [5] has presented results for the case of disks impulsively set in motion with constant angular velocities at time $t=0$. For $s=-1$ and $R_{e}^{R}=100$ he obtained an azimuthal velocity profile which was at all times symmetrical about the mid-plane between the disks. But at $s=-1, R_{e}^{R}=1000$ he obtained a non-symmetrical solution which started to develop at small $t$; it is possible, therefore, that the solution to the governing equations is not unique for any value of $t>0$ when $s=-1$ and $R_{e}^{R}=1000$.

It is very likely that for sufficiently large $R_{e}^{R}$ and $\left(R_{e}^{R} / R_{e}^{s}\right)$ Eqs. (2.7)-(2.10) of the present paper will present similar difficulties. But we have no evidence to suggest that their solution is other than unique when $R_{e}^{s}>0$ and $0 \leqslant R_{e}^{R} \leqslant 100$.

\section{REFERENCES}

[1] Th. v. Kármán, Z. Angew. Math. Mech. 1 (1921), 244

[2] G. K. Batchelor, Quart. J. Mech. Appl. Math. 4 (1951), 29

[3] K. Stewartson, Proc. Camb. Phil. Soc. 49 (1953), 333

[4] M. Holodniok, M. Kubičk and V. Hlaváček, J. Fluid Mech. 108 (1981), 227

[5] C. E. Pearson, J. Fluid Mech. 21 (1965), 623

[6] S. Ishizawa, Bull. J.S.M.E. 9 (1966), 533

[7] C. Y. Wang, J. Apl. Mech., Trans. A.S.M.E., 43 (1976), 579

[8] W. G. Cochran, Proc. Camb. Phil. Soc. 30 (1934), 365

[9] G. N. Lance and M. H. Rogers, Proc. Roy. Soc. (A) 266 (1962), 109

[10] M. Holodniok, M. Kubiček and V. Hlaváček, J. Fluid Mech. 81 (1977), 689 